\title{
LONG PERIOD TERMS IN THE SOLAR SYSTEM
}

\author{
P. BRETAGNON \\ Bureau des Longitudes, Paris, France
}

L'ensemble de ce travail fera l'objet d'une publication dans Astronomy and Astrophysics.

Nous avons étudié les variations à longues périodes des éléments des huit planètes du système solaire. Pour cela, nous avons calculé la solution de Lagrange puis introduit les termes d'ordre 4 à longues périodes de la fonction perturbatrice ainsi que la contribution, au premier ordre des masses, des termes à courtes périodes. Nous nous sommes, de plus, tout particulièrement attachés au problème de la détermination des constantes d'intégration. En effet, nous avons obtenu une grande modification des constantes d'intégration en particulier pour les constantes relatives aux planètes Mercure, Vénus, Terre et Mars. Ceci provient de l'importance, pour ces planètes, des termes d'ordre 3. La contribution des termes à courtes périodes apporte surtout une modification des fréquences relatives aux planètes Jupiter, Saturne, Uranus et Neptune.

Ce travail montre donc l'importance relative des différentes contributions: il est, par exemple, inutile d'introduire les termes d'ordre 5 à longues périodes si on n'a pas tenu compte des termes à courtes périodes. 